\section{Einfluss einer nicht sterilen Umgebung}

\section{Können schwer granulozytopene Patienten ein Kinder-Sommercamp ohne zusätzliches Risiko einer Infektion aufsuchen? Ein erster Versuch verlief durchaus ermutigend.}

K anadische Autoren evaluierten das Komplikationsrisiko von Kindern, die während einer Phase mit schwerer Granulozytopenie ein von der behandelnden Klinik organisiertes Sommercamp besuchten. Die Studie wurde retrospektiv durchgeführt. Die 34 untersuchten Kinder litten zum Zeitpunkt der Untersuchung an einer schweren Granulozytopenie (definiert als Granulozytenzahl $\leq 500 / \mu \mathrm{l})$ oder befanden sich in der intensiven Phase der Chemotherapie. Die analysierten Endpunkte umfassten Fieber, Blutungskomplikationen, Hospitalisierung und den laborchemischen Hinweis auf eine Infektion.

Obwohl von diesen Kindern neun (24\%) aufgrund von Fieber bei Granulozytopenie im Krankenhaus aufgenommen und behandelt werden mussten, konnte nur bei einem klinisch und mikrobiolo- gisch eine Infektion nachgewiesen werden. Bei keinem Patienten ereigneten sich Blutungskomplikationen, obwohl alle analysierten Patienten an praktisch allen Aktivitäten des Camps teilnahmen.

Die Autoren schlussfolgern, dass auch schwer granulozytopene Patienten eine nicht sterile Umgebung wie ein Sommercamp ohne zusätzliches Risiko einer Infektion aufsuchen können. Weiterhin folgern sie, dass die Ergebnisse möglicherweise auf Schule und andere soziale Aktivitäten übertragen werden könnten.

Kommentar: Insgesamt ist es sehr begrüßenswert, dass sich die Autoren an eine kritische Überarbeitung der etablierten „Schutzstandards“ für krebskranke Kinder wagen. Die erste Schlussfolgerung der Autoren, dass granulozytopene Pati- enten ein von der Klinik organisiertes Sommercamp ohne zusätzliches Infektionsrisiko besuchen können, ist meines Erachtens richtig. Allerdings können die Ergebnisse nicht ohne weiteres auf andere Settings übertragen werden, da das Sommercamp im Gegensatz zu vielen anderen Orten wesentlich geschützter ist. Dies bezieht sich insbesondere auf den Schutz vor Personen mit ansteckenden Erkrankungen, die sicherlich durch das ärztliche Personal nicht auf das Camp gelassen werden, vor denen Patienten in öffentlichen Verkehrmitteln oder Gebäuden jedoch nicht geschützt sind. Auch können aufgrund der geringen Anzahl der Patienten wenig Aussagen zu Untergruppen gemacht werden, da auch andere Nebenwirkungen der Chemotherapie, wie z. B. eine begleitende Mukositis, die Infektionsgefahr bei Patienten weiter erhöht. Prof. Dr. Thomas Lehrnbecher

Tabori $U$ et al. Low prevalence of complications in severe neutropenic children with cancer in the unprotected environment of an overnight camp. Pediatr Blood Cancer 2007; 48: 148-51

\title{
Ohne Compliance keine effektive Prävention
}

\section{Eine deutsche Studie sollte klären, inwieweit Maßnahmen zur Infektions- vermeidung von den Patienten auch tatsächlich umgesetzt werden.}

Antimykotika $(88,2 \%)$ und den Einsatz von Trimethoprim und Sulfametoxazol $(86,6 \%)$ ermittelt. Am niedrigsten war die Compliance beim Gebrauch eines Mundschutzes $(68,8 \%)$, bei antiseptischen Mundspülungen (67,1\%), nicht absorbierbaren Antibiotika (66,5\%) und Restriktionen im sozialen Kontakt (65,5\%). Die häufigsten Gründe für eine NonCompliance waren Vergesslichkeit und fehlende Akzeptanz für die Maßnahme. Die Compliance korrelierte mit der Diagnose einer hämatologischen Erkrankung, jüngerem Alter und dem Glauben an die Effektivität, aber nicht mit der individuell empfundenen Belastung durch die Maßnahme oder der Zufriedenheit des Patienten bzw. dessen Eltern.

Die Autoren folgern aus diesen Ergebnissen, dass die Compliance mit prophy- laktischen antiinfektiösen Maßnahem sehr variabel ist und unter anderem mit dem Glauben an deren Effektivität zusammenhängt.

Kommentar: Diese Untersuchung ist einer der wenigen publizierten Versuche, Hintergründe für die Compliance und Non-Compliance zu erfassen. Die Untersuchung zeigt, dass auch bei Kindern mit Krebserkrankungen Compliance nicht automatisch vorausgesetzt werden kann. Sie zeigt außerdem, dass weitere Studien notwendig sind, um zu eruieren, bei welchen Patienten Compliance angenommen werden kann bzw. bei welchen sie gefördert werden müsste. Dies würde möglicherweise einen nachhaltigen Effekt auf die Effektivität einer Maßnahme haben.

Prof. Dr. Thomas Lehrnbecher

Lehrnbecher T et al. Compliance with antiinfective preventive measures: a multicentre survey among paediatric oncology patients. Eur J Cancer 2008; 44: 1861-5 


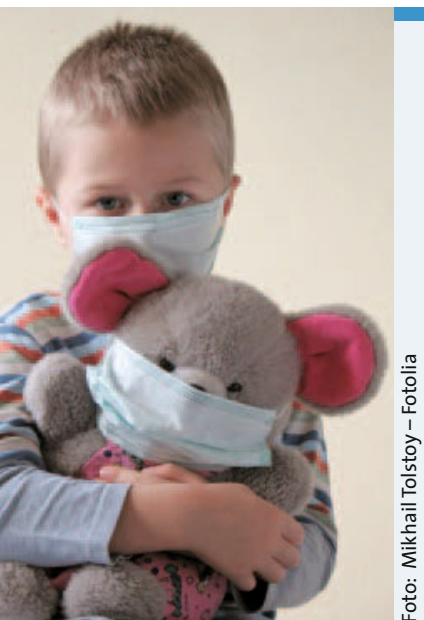

\section{Infektionen bei Kindern mit Krebserkrankungen}

Infektiöse Komplikationen sind eine wichtige und oft lebensgefährliche Nebenwirkung der Chemotherapie bei Kindern mit Krebserkrankungen. Drei Literaturreferate beleuchten auf dieser Doppelseite das Thema von verschiedenen Seiten.

Je nach Grunderkrankung sterben bis zu $10 \%$ der Kinder während der Therapie aufgrund von Infektionen. Kinder mit akuter myeloischer Leukämie erleiden während der etwa sechsmonatigen Intensivphase der Chemotherapie im Schnitt drei infek-

: tiöse Komplikationen, die neben der Hospitalisierung auch teure diagnostische und therapeutische Maßnahmen nach sich ziehen. Zudem verzögern Infektionen oft die Chemotherapie- und Bestrahlungseinheiten und führen so möglicherweise zu einer Verschlechterung der Heilungschancen.

Aus diesem Grund wurden gerade in der Anfangsphase der Kinderhämatologie und -onkologie in den 6oer Jahren Strategien zur Prävention von Infektionen entwickelt. So sollten nicht medikamentöse Maßnahmen wie die Vermeidung von sozialen Kontakten oder auch das Tragen eines Mundschutzes beim Verlassen des Hauses als Art Expositionsprophylaxe dienen. Darüber hinaus wurde versucht, mit einer medikamentösen Prophylaxe wie antiseptischen Mundspülungen oder nicht absorbierbaren Antibiotika das Risiko einer Infektion zu vermindern. All diese Maßnahmen haben dazu geführt, dass die Letalität durch Infektionen deutlich gesunken ist. Allerdings hat sich über die letzten Jahrzehnte vieles verändert, weswegen man möglicherweise die etablierten Prophylaxestrategien überdenken sollte. So wurde die Therapieintensität in der Kinderonkologie für „Standardrisikopatienten“ reduziert, die die Mehrzahl der Patienten stellt, für nur eine einzige Untergruppe wurden sie erhöht.

Wie bei den Therapieregimen wissen wir heutzutage auch wesentlich besser das individuelle Risiko für eine schwere Infektion abzuschätzen. Daher müssen die behandelnden Ärzte regelmäßig analysieren, wie viele Patienten mit welchen Grunderkrankungen an einer Infektion sterben und durch welche Erreger diese Infektionen verursacht wurden. Dies hat Einfluss auf die gewählten Prophylaxestrategien, wobei auch der Sinn und die Effektivität etablierter Maßnahmen immer wieder kritisch hinterfragt werden müssen. Letztendlich sollte auch geprüft werden, inwiefern die Patienten die ihnen angeratenen Maßnahmen überhaupt befolgen.

Alle drei vorliegenden Studien zeigen letztlich, dass es wichtig ist, die Inzidenz und den Verlauf von Infektionen genauer zu analysieren, um entscheidende Faktoren zu finden, die für einen letalen Ausgang verantwortlich sind. Gleichzeitig müssen viele bisher als sinnvoll angenommenen Maßnahmen kritisch überdacht und gegebenenfalls neu überprüft werden. Es muss aber auch untersucht werden, inwieweit die Prophylaxe von den Patienten überhaupt angenommen wird. Um diese Untersuchungen an einem großen, möglichst homogenen pädiatrischen Patientengut durchführen zu können, sind internationale Kooperationen notwendig.

Prof. Dr. Thomas Lehrnbecher

\section{Todesraten nach Infektionen}

\section{Eine britische Publikation liefert aktuelle Zahlen zur Häufigkeit von infektionsbedingten Todesfällen bei krebskranken Kindern.}

Eine ine Studie aus England und Wales analysierte infektionsassoziierte Todesfälle bei Kindern, die in einem Alter unter 15 Jahren zwischen 2003 und 2005 verstorben waren. Alle hatten an einer hämatologischen Grunderkrankung oder an einem soliden Tumor gelitten.

Die Autoren identifizierten unter diesen Kriterien 82 Fälle, was insgesamt 2\% aller an Krebs erkrankten Kinder ausmachte. Die Infektionen hatten etwa $25 \%$ der Todesfälle bei den hämatologischen Erkrankungen zur Folge und 5\% bei den soliden Tumoren. Unter den 82 Patienten befanden sich 15 (18\%) mit einem Rezidiv, fünf $(6 \%)$ nach hämatopoetischer Stammzelltransplantation und vier (5\%) mit Down-Syndrom. Bei lediglich 54\% konnte ein ursächlicher
Erreger gefunden werden: 17 Patienten (21\%) starben aufgrund einer invasiven Pilzinfektion, davon zwölf (15\%) durch Aspergillus, 17 (21\%) durch eine bakterielle Infektion, davon neun (11\%) durch Gram-negative Bakterien. Insgesamt zehn der Kinder (12\%) starben durch Virus-assoziierte Komplikationen, wobei bei drei Kindern das Respiratory Syncytial Virus gefunden wurde.

Die Autoren folgern, dass Infektionen auch in der heutigen Zeit eine signifikante Gefahr für Kinder mit Krebserkrankungen darstellen und dass effektive Präventionsmaßnahmen identifiziert werden müssen.

Kommentar: Die Publikation zeigt, dass infektiöse Komplikationen immer noch eine tödliche Gefahr für krebskranke Kinder sind, insbesondere für Kinder mit einer hämatologischen Grunderkrankung, weniger für Kinder mit einem soliden Tumor. Allerdings zeigt sich, dass die Zahl der analysierten Todesfälle insgesamt gering ist. Darüber hinaus fehlt in der Arbeit eine genaue Analyse der prophylaktischen Maßnahmen sowie der Umstände, unter denen die Patienten verstarben. Nur so könnten aber letztendlich Aussagen darüber getroffen werden, ob prophylaktische Maßnahmen möglicherweise den Tod verhindert hätten. Hier wären internationale Kooperationen notwendig, mit denen deutlich mehr Patienten und Komplikationen erfasst werden könnten.

Prof. Dr. Thomas Lehrnbecher

Bate J et al. Infection-related mortality in children with malignancy in England and Wales, 2003-2005. Pediatr Blood Cancer 2009; 53: 371-4 